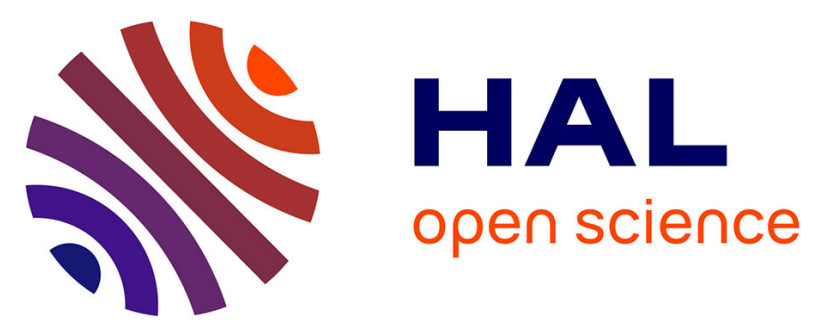

\title{
Catalytic activity in transfer hydrogenation using ruthenium (II) carbonyl complexes containing two 1,8-naphthyridine as $\mathrm{N}$-monodentate ligands
}

J. Gajardo, J.C. Araya, Andres Ibanez, V. Guerchais, H. Le Bozec, S.A. Moya, P. Aguirre

\section{To cite this version:}

J. Gajardo, J.C. Araya, Andres Ibanez, V. Guerchais, H. Le Bozec, et al.. Catalytic activity in transfer hydrogenation using ruthenium (II) carbonyl complexes containing two 1,8-naphthyridine as N-monodentate ligands. Inorganica Chimica Acta, 2019, 486, pp.129-134. 10.1016/j.ica.2018.10.037 . hal-01939030

\section{HAL Id: hal-01939030 \\ https://hal-univ-rennes1.archives-ouvertes.fr/hal-01939030}

Submitted on 13 Dec 2018

HAL is a multi-disciplinary open access archive for the deposit and dissemination of scientific research documents, whether they are published or not. The documents may come from teaching and research institutions in France or abroad, or from public or private research centers.
L'archive ouverte pluridisciplinaire HAL, est destinée au dépôt et à la diffusion de documents scientifiques de niveau recherche, publiés ou non, émanant des établissements d'enseignement et de recherche français ou étrangers, des laboratoires publics ou privés. 


\section{Catalytic activity in transfer hydrogenation using ruthenium (II) carbonyl complexes containing two 1,8-naphthyridine as $\mathrm{N}$-monodentate ligands.}

Juana Gajardo ${ }^{a}$, Juan C. Araya ${ }^{b}$, Andrés Ibáñez $^{c}$, Véronique Guerchais ${ }^{d}$, Hubert Le Bozec $^{d}$, Sergio A. Moya ${ }^{* e}$ and Pedro Aguirre ${ }^{* f}$

aUniversidad San Sebastián, Facultad de Ciencias, Depto. de Ciencias Biológicas y Químicas, Santiago, Chile.

bUniversidad Central, Centro de Ciencias Básicas, Facultad de Ciencias de la Salud, Santiago, Chile.

'Universidad de Chile, Facultad de Ciencias Físicas y Matemáticas, Santiago, Chile.

dUMR 6226 CNRS-Université de Rennes 1, Sciences Chimiques de Rennes, Campus de Beaulieu, 35042 Rennes, France.

eUniversidad de Santiago de Chile, Facultad de Química y Biología, Avda. Libertador Bernardo O'Higgins 3363, casilla 40, correo 33, Santiago, Chile. sergio.moya@usach.cl

fUniversidad de Chile, Facultad de Ciencias Químicas y Farmacéuticas, Santiago, Chile.paguirre@ciq.uchile.cl

This manuscript is dedicated to Professor Hubert Le Bozec from Rennes University, France, who retired recently. Professor Le Bozec had a long and fruitful collaboration with our group in Chile for 30 years. During that time many Chilean postgraduate students made part of their theses in the University of Rennes, supervised by Prof. Le Bozec. Throughout his collaboration with us, we have not only an excellent co-worker but also a great friend and we are sure that our friendship will last forever.

\section{Abstract}

A new series of novel complexes of type cis- $\left[\mathrm{Ru}(\mathrm{CO})_{2} \mathrm{Cl}_{2}(\mathrm{~L})_{2}\right], \quad \mathrm{L}=2$-phenyl-1,8naphthyridine, 2-(4'-nitrophenyl)-1,8-naphthyridine, 2-(4'bromophenyl)-1,8naphthyridine, 2-(4'-methylphenyl)-1,8-naphthyridine, 2-(3'-methoxyphenyl)-1,8- 
naphthyridine, 2-(2'-methoxyphenyl)-1,8-naphthyridine and 2-(4'-methoxyphenyl)1,8-naphthyridine have been successfully synthesized and characterized. We found that the complexes can be directly synthesized from $\left[\mathrm{RuCl}_{2}(\mathrm{CO})_{2}\right]_{2}$ with high yield. The crystallographic structures of complex cis- $\left[\mathrm{RuCl}_{2}(\mathrm{CO})_{2}(2-(4)-\right.$ methoxyphenyl)-1,8-naphthyridine-kN8 $\left.)_{2}\right]$ and $\quad$ cis- $\left[\mathrm{RuCl}_{2}(\mathrm{CO})_{2}(2-(2 '-\right.$ methoxyphenyl)-1,8-naphthyridine-kN8 ${ }_{2}$ ] have been established by $\mathrm{X}$-Ray single crystal diffraction studies, which indicate an octahedral geometry with two 1,8naphthyridine ligands coordinated to the metal in a $\mathbf{N}$-monodentate fashion. The ruthenium(II) complexes have been studied as catalysts in the transfer hydrogenation of acetophenone. We found that complexes show moderate activities and a $100 \%$ selectivity. The best turnover frequency $\left(390 \mathrm{~h}^{-1}\right)$ is found for cis-[RuCl $2(\mathrm{CO})_{2}\left(2-\left(4^{\prime} \text {-methoxyphenyl)-1,8-naphthyridine-kN8 }\right)_{2}\right]$ when the substrate/catalysis ratio was $1000 / 1$. The catalytic conditions were optimized using different substrate/catalyst and base/catalyst ratios.

Keywords: Ruthenium (II), 1,8-naphthyridine complexes, transfer hydrogenation, acetophenone, homogeneous catalysis.

\section{Introduction}

The naphthyridines (NAPs) are a group of diazanaphthalenes that contain a single nitrogen atom in each ring but no nitrogen atom at either of the bridgehead positions [1]. Several structural isomers exist, where the 1,8-NAPs are key components of a number of antibacterial agents [2]. The synthetic procedure are prepared via the Friedländer condensation of 2-aminonicotinaldehyde with the corresponding acyl derivatives[3-6]. In this system, the precursor 2aminonicotinaldehyde must be freshly prepared and used immediately after isolation to avoid self-condensation side reactions. Indeed, a facile synthesis of 2aminonicotinaldehyde was reported by Caluwe and co-workers in 1974 [5], with modifications being introduced later by Dunbar et all.,Rivera et al. [7, 8]. In addition, the preparation of various alkylsubstituted (i.e., 2- and/or 3-position) NAP ligands has also been reported [9-14]. During the formation of mononuclear 
ruthenium complexes, 1,8-NAP (1) tends to act as either a monodentate or a bidentate ligand. However, chelation is generally disfavored due to the small bite angle of the four-membered chelate ring.

Ruthenium complexes derived from 1,8-NAP have also been applied in homogeneous catalytic reactions as substitutes for 2,2-bipyridine or 1,10phenanthroline (phen). For example, the catalytic potential of ruthenium complexes containing monodentate 1,8-NAPs, was investigated in the hydroformylation of styrene in dimethylformamide [15].

Ruthenium arene complexes, $\left[\left(\eta^{6}-\mathrm{p} \text {-cymene }\right)_{2} \mathrm{Ru}_{2}(\mathrm{~L}) \mathrm{Cl}_{2}\right]\left(\mathrm{PF}_{6}\right)_{2} \mathrm{~L}=7$-bis(di-2pyridinyl)-1,8- naphthyridine] was synthesized and characterized by spectroscopic and analytical techniques. The use of these ruthenium complexes as pre-catalysts for oxidative coupling of 1,2-diols/1,2-aminoalcohol with o-phenylenediamines leading to quinoxalines was investigated. [16]. Other ruthenium complexes containing 1,8 NAP derivate have been succefully prepared and used as anticarcer compunds[ 17]

Other authors found that ruthenium-hydride complexes containing 1,8 NaPs ligands are effective catalysts in the transformation of primary alcohols, including amino alcohols, into the corresponding carboxylic acid in the presence of alkaline water [18]. This work proposed that the 1,8-NAP unit enhances the nucleophilicity of a water molecule through hydrogen bonding, and that the noncoordinated nitrogen atom in the 1,8-NAP unit promotes solvated hydroxide attack through hydrogen bonding with a water molecule of the solvent cage. Alternatively, uncoordinated nitrogen atom may facilitate hydroxide attack by coordination with $\mathrm{Na}^{+}$.

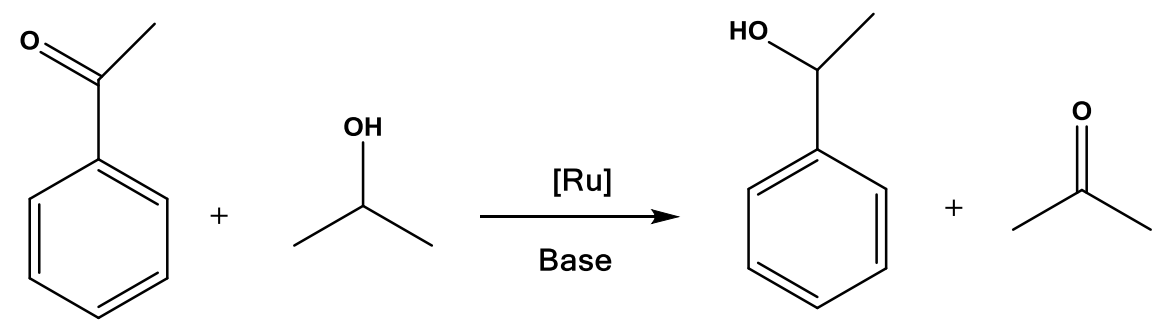


Scheme 1. Hydrogen transfer reaction of ketones (HTR).

Transfer hydrogenation of ketones by propan-2-ol is convenient in large-scale synthesis since there is no need to employ a high hydrogen pressure or to use hazardous reducing agents (scheme 1). Transition metal complexes containing a coordinative unsaturated metal center and a Brønsted basic $\pi$-donor ligand have been reported in homogeneous transfer hydrogenation. Ligands with different transition metals such as amido [19-22], thiolate [23-28], phosphido [29], imido [3040], oxo [41-46], and sulfido [47] have been investigated for this reaction. The most active and selective catalysts for the transfer hydrogenation reactions are ruthenium [48], iridium [49] and rhodium [50] complexes containing nitrogen, oxygen and N,O-donor ligands [51-58]. We have investigated the synthesis of ruthenium complexes containing polypyridine ligands and their application as potential homogeneous catalysts. We found that the compounds are active in the transfer hydrogenation where the active species is a ruthenium hydride complex containing 2,2-bipyridine, phenanthroline and 1,8-naphthyridine ligands.

Herein, we report the synthesis and the catalytic behavior of ruthenium complexes of the type cis- $\left[\mathrm{Ru}(\mathrm{CO})_{2} \mathrm{Cl}_{2}(\mathbf{L})_{2}\right]$ with $\mathbf{L}=2$-phenyl-1,8-naphthyridine; $\mathbf{L}_{\mathbf{1}}$, 2-(4'nitrophenyl)-1,8-naphthyridine; , $L_{2}, 2$ (4'-bromophenyl)-1,8-naphthyridine; , $L_{3}, \quad 2$ (4'-methylphenyl)1,8-naphthyridine; , L $\mathbf{L}_{\mathbf{4}}, 2$-(4'-methoxyphenyl)-1,8-naphthyridine; , $\mathbf{L}_{5}, \quad$ 2-(3'-methoxyphenyl)-1,8-naphthyridine;; , $\mathbf{L}_{6}$ and 2-(2'-methoxyphenyl)-1,8naphthyridine, $\mathbf{L}_{\mathbf{7}}$, in hydrogenation reaction of ketones. The X-Ray structures of complexes show that two naphthyridines ligands coordinate in an $\mathrm{N}$-monodentate fashion to the metal. We will describe detailed studies on their catalytic activities for the hydrogen transfer reaction of ketones (HTR).

\section{Experimental part.}

\section{Materials and general methods}

All the reagents used were chemically pure and analytical grade. Acetophenone was used as supplied from Sigma-Aldrich. $\left[\mathrm{RuCl}_{2}(\mathrm{CO})_{2}\right]_{2}$ [59] was prepared by refluxing commercial $\mathrm{RuCl}_{3} \cdot 3 \mathrm{H}_{2} \mathrm{O}$ in a mixture of $37 \%$ hydrochloric acid and $88 \%$ formic acid (1:1) during 24 hours under nitrogen. The ligands were 
prepared from 2-aminonicotinaldehyde and substituted acetophenone. The substrates were obtained from Sigma-Aldrich.

The catalytic conversions were determinated by gas chromatography using an Agilent 6890 Series GC System equipped with a flame ionization detector and a (30 m・0.25 mm・ $0.25 \mu \mathrm{m}$ ) HP-INNOWAX column.

Crystal structure analysis of complexes. The data were collected at a temperature of $293 \pm 2^{\circ} \mathrm{K}$ on an Oxford Diffraction X calibur Saphir 3 diffractometer equipped with graphite monochromated MoKa radiation (=0.71069 $\AA$ ). The structure was solved with SIR-97, which reveals the non-hydrogen atoms of the molecules. The structure was solved in the space group $P 2_{1} / C$ by Patterson or direct method and refined by the full-matrix least-squares fitting on $F^{2}$ using SHELXTL-97 with initial isotropic parameters.

\section{General procedure Synthesis of ligands}

A suspension of 2-aminonicotinaldehyde (500 $\mathrm{mg} ; 4.09 \mathrm{mmol})$, substituted acetophenone (4.09 mmol), and $\mathrm{NaOH}(200 \mathrm{mg} ; 5 \mathrm{mmol})$ in $\mathrm{EtOH}(40 \mathrm{~mL})$ was refluxed under argon for 4 hours. The solvent was removed under vacuum. The residue was extracted from a mixture of $\mathrm{CH}_{2} \mathrm{Cl}_{2}$ /water and the organic layer was dried over magnesium sulfate; the product was purified by column chromatography on silica gel eluting with $\mathrm{CH}_{2} \mathrm{Cl}_{2}$ :EtOAc (6:1). After the solvent was removed, the desired product was obtained as a white solid. (more information about ligands characterization see supplemental material) .

\section{Synthesis of complexes}

The ligands $\mathbf{L}(1.316 \mathrm{mmol})$ and $\left[\mathrm{RuCl}_{2}(\mathrm{CO})_{2}\right]_{2}(0.658 \mathrm{mmol})$ were mixed in ethanol/ $\mathrm{H}_{2} \mathrm{O}(9 / 1)(50 \mathrm{~mL})$ The mixture was refluxed for 24 hours under nitrogen atmosphere. The precipitate was separated by filtration and the solid was washed with, ethanol, acetone, chloroform and ether ethylic.

Cis-[RuCl ${ }_{2}(\mathrm{CO})_{2}$ (2-phenyl-1,8-naphthyridine-k N8) $)_{2}(\mathrm{Ru}-1)$.

${ }^{1} \mathrm{H}-\mathrm{NMR}\left(200 \mathrm{MHz} \mathrm{CDCl}_{3}\right) 10.44$ (dd, $\left.\mathrm{H}_{7}, \mathrm{~J}=5.4 \mathrm{~Hz}, \mathrm{~J}=2.0 \mathrm{~Hz}, 1 \mathrm{H}\right), 8.98$ (dd, $\mathrm{H}_{7}$, $\mathrm{J}=5.3 \mathrm{~Hz}, 1.9 \mathrm{~Hz}, 1 \mathrm{H}), 8.83\left(\mathrm{dd}, \mathrm{H}_{5}, \mathrm{~J}=8.0 \mathrm{~Hz}, \mathrm{~J}=2.0 \mathrm{~Hz}, 1 \mathrm{H}\right), 8.75(\mathrm{~m}, \mathrm{o}-\mathrm{Ph}, \mathrm{m}-$ $\mathrm{Ph}, 2 \mathrm{H}), 8.65$ (d, $\left.\mathrm{H}_{4}, \mathrm{~J}=8.4 \mathrm{~Hz}, 1 \mathrm{H}\right), 8.45\left(\mathrm{~d}, \mathrm{H}_{4}, \mathrm{~J}=8.8 \mathrm{~Hz}, 1 \mathrm{H}\right), 8.43\left(\mathrm{~d}, \mathrm{H}_{3^{\prime}}, \mathrm{J}=\right.$ $8.8 \mathrm{~Hz}, 1 \mathrm{H}), 8.29\left(\mathrm{dd}, \mathrm{H}_{5}, \mathrm{~J}=8.0 \mathrm{~Hz}, \mathrm{~J}=1.9 \mathrm{~Hz}, 1 \mathrm{H}\right), 8.10\left(\mathrm{~d}, \mathrm{H}_{3}, \mathrm{~J}=8.4 \mathrm{~Hz}, 1 \mathrm{H}\right)$, $8.01\left(\mathrm{dd}, \mathrm{H}_{6}, \mathrm{~J}=8.0 \mathrm{~Hz}, \mathrm{~J}=5.4 \mathrm{~Hz}, 1 \mathrm{H}\right), 7.78(\mathrm{~m}, \mathrm{~m}-\mathrm{Ph}, 1 \mathrm{H}), 7.70(\mathrm{~m}, \mathrm{p}-\mathrm{Ph}, 1 \mathrm{H})$, 7.37 (m, m-Ph, 1H), $7.35(\mathrm{~m}, 0-\mathrm{Ph}, 1 \mathrm{H}), 7.24(\mathrm{~m}, 0-\mathrm{Ph}, 1 \mathrm{H}), 7.09\left(\mathrm{dd}, \mathrm{H}_{6}, \mathrm{~J}=8.0\right.$ $\mathrm{Hz}, \mathrm{J}=5.3 \mathrm{~Hz}, 1 \mathrm{H}), 6.95(\mathrm{~m}, \mathrm{o}-\mathrm{Ph}, 1 \mathrm{H})$. Yield $65 \%$. MP $340{ }^{\circ} \mathrm{C}(\mathrm{d}) . \mathrm{IR}\left(\mathrm{KBr}, \mathrm{cm}^{-1}\right)$ 
M-CO 2044.7; 1983.2. Anal. Calcd. (\%) for: $\mathrm{C}_{30} \mathrm{H}_{20} \mathrm{Cl}_{2} \mathrm{~N}_{4} \mathrm{O}_{2} \mathrm{Ru} \mathrm{C}, 56.26 ; \mathrm{H}, 3.15 ; \mathrm{N}$, 8.75. Found (\%): C, 55.95; H, 2.95; N, 8.76.

\section{Cis-[RuCl $\left.{ }_{2}(\mathrm{CO})_{2}(2-(4 '-n i t r o p h e n y l)-1,8-n a p h t h y r i d i n e-k N 8)_{2}\right](\mathbf{R u}-2)$.}

${ }^{1} \mathrm{H}-\mathrm{NMR}\left(200 \mathrm{MHz}, \mathrm{CDCl}_{3}\right) 10.93$ (dd, $\left.\mathrm{H}_{7}, \mathrm{~J}=5.3 \mathrm{~Hz}, \mathrm{~J}=1.8 \mathrm{~Hz}, 1 \mathrm{H}\right), 8.98\left(\mathrm{~m}, \mathrm{H}_{7}\right.$, $\mathrm{Ph}^{\prime} 3 \mathrm{H}$ ), 8.91 (dd, $\left.\mathrm{H}_{5}, \mathrm{~J}=8.1, \mathrm{~J}=1.8,1 \mathrm{H}\right), 8.71(\mathrm{~m}, 2 \mathrm{H}, \mathrm{Ph}), 8.69\left(\mathrm{~d}, \mathrm{H}_{4}, \mathrm{~J}=8.4\right.$ $\mathrm{Hz}, 1 \mathrm{H}), 8.56\left(\mathrm{~d}, \mathrm{H}_{4}, \mathrm{~J}=8.6 \mathrm{~Hz}, 1 \mathrm{H}\right), 8.49\left(\mathrm{~d}, \mathrm{H}_{3^{\prime}}, \mathrm{J}=8.6,1 \mathrm{H}\right), 8.32\left(\mathrm{dd}, \mathrm{H}_{5}, \mathrm{~J}=8.0\right.$ $\mathrm{Hz}, \mathrm{J}=1.6 \mathrm{~Hz}, 1 \mathrm{H}), 8.15\left(\mathrm{~d}, \mathrm{H}_{3}, \mathrm{~J}=8.4 \mathrm{~Hz}, 1 \mathrm{H}\right), 8.1\left(\mathrm{dd}, \mathrm{H}_{6}, \mathrm{~J}=8.1, \mathrm{~J}=5.3,1 \mathrm{H}\right)$, 7.82 (m. Ph', 2H), 7.57 (m, Ph, 2H), 7.09 (dd, $\mathrm{H}_{6^{\prime}}, \mathrm{J}=8.0 \mathrm{~Hz}, \mathrm{~J}=5.2 \mathrm{~Hz}, 1 \mathrm{H}$ ). Yield $57 \%$. MP $328{ }^{\circ} \mathrm{C}(\mathrm{d})$. IR $\left(\mathrm{KBr}, \mathrm{cm}^{-1}\right)$ : M-CO 2051.6; 1987.6. Anal. Calcd. (\%) for $\mathrm{C}_{30} \mathrm{H}_{18} \mathrm{Cl}_{2} \mathrm{~N}_{6} \mathrm{O}_{6} \mathrm{Ru}: \mathrm{C}, 49.33 ; \mathrm{H}, 2.48 ; \mathrm{N}, 11.50$. Found (\%): C, 48.96; $\mathrm{H}, 2.32 ; \mathrm{N}$, 11.26 .

\section{Cis-[RuCl${ }_{2}(\mathrm{CO})_{2}$ (2-(4'-bromophenyl)-1,8-naphthyridine-kN8) $)_{2}$ (Ru-3).}

${ }^{1} \mathrm{H}-\mathrm{NMR}\left(200 \mathrm{MHz}, \mathrm{CDCl}_{3}\right) 10.05$ (dd, $\left.\mathrm{H}_{7}, \mathrm{~J}=5.4 \mathrm{~Hz}, \mathrm{~J}=1.8 \mathrm{~Hz}, 1 \mathrm{H}\right), 9.76\left(\mathrm{dd}, \mathrm{H}_{7}\right.$, $\mathrm{J}=5.4 \mathrm{~Hz}, \mathrm{~J}=1.8 \mathrm{~Hz}, 1 \mathrm{H}), 8.52\left(\mathrm{dd}, \mathrm{H}_{5}, \mathrm{~J}=8.1 \mathrm{~Hz}, \mathrm{~J}=1.8 \mathrm{~Hz}, 1 \mathrm{H}\right), 8.47\left(\mathrm{~d}, \mathrm{H}_{3}, \mathrm{~J}\right.$ $=8.6 \mathrm{~Hz}, 1 \mathrm{H}), 8.36\left(\mathrm{dd}, \mathrm{H}_{5}, \mathrm{~J}=8.1 \mathrm{~Hz}, 1.8 \mathrm{~Hz}, 1 \mathrm{H}\right), 8.35\left(\mathrm{~d}, \mathrm{H}_{4}, \mathrm{~J}=8.9 \mathrm{~Hz}, 1 \mathrm{H}\right)$, 8.17(d, $\left.\mathrm{H}_{3}, \mathrm{~J}=8.6 \mathrm{~Hz}, 1 \mathrm{H}\right), 8.09\left(\mathrm{~d}, \mathrm{H}_{3}, \mathrm{~J}=8.9 \mathrm{~Hz}, 1 \mathrm{H}\right), 8.05\left(\mathrm{~m}, \mathrm{Ph}, \mathrm{Ph}^{\prime}, 3 \mathrm{H}\right)$, 7.82 (dd, $\mathrm{H}_{6}, \mathrm{~J}=8.1 \mathrm{~Hz}, \mathrm{~J}=5.4 \mathrm{~Hz}$ ), 7.79 (d, Ph', J = 8.7 Hz), 2H), 7.64 (dd. $\mathrm{H}_{6^{\prime}}$, J $=8.1 \mathrm{~Hz}, \mathrm{~J}=5.4 \mathrm{~Hz})$. Yield 67\%. MP $368^{\circ} \mathrm{C}(\mathrm{d}) . \mathrm{IR}\left(\mathrm{KBr}, \mathrm{cm}^{-1}\right) \mathrm{M}-\mathrm{CO} 2050.2 ;$ 1985.6. Calcd. (\%) for $\mathrm{C}_{30} \mathrm{H}_{18} \mathrm{Br}_{2} \mathrm{~N}_{4} \mathrm{O}_{2} \mathrm{Ru}$ : C, 45.14; $\mathrm{H}, 2.27 ; \mathrm{N}, 7.02$. Found (\%): C, 44.96; H, 2.37; N, 6.79 .

\section{Cis-[RuCl${ }_{2}(\mathrm{CO})_{2}$ (2-(4'-methylphenyl)-1,8-naphthyridine- $\left.\left.\mathrm{N} 8\right)_{2}\right](\mathrm{Ru}-4)$.}

${ }^{1} \mathrm{H}-\mathrm{NMR}\left(200 \mathrm{MHz}, \mathrm{CDCl}_{3}\right) 10.47$ (dd, $\left.\mathrm{H}_{7}, \mathrm{~J}=5.5 \mathrm{~Hz}, \mathrm{~J}=1.9 \mathrm{~Hz}, 1 \mathrm{H}\right), 8.85$ (dd, $\mathrm{H}_{7}$, $\mathrm{J}=5.4 \mathrm{~Hz}, \mathrm{~J}=1.0 \mathrm{~Hz}), 8.46(\mathrm{~d}, \mathrm{Ph}, 1 \mathrm{H}), 8.38\left(\mathrm{dd}, \mathrm{H}_{5}, \mathrm{~J}=7.8 \mathrm{~Hz}, \mathrm{~J}=1.9 \mathrm{~Hz}, 1 \mathrm{H}\right)$, $8.20\left(\mathrm{~d}, \mathrm{H}_{4}, \mathrm{H}_{4}, \mathrm{~J}=8.4 \mathrm{~Hz}, 2 \mathrm{H}\right), 8.05\left(\mathrm{~d}, \mathrm{H}_{3}, \mathrm{~J}=8.5 \mathrm{~Hz}, 1 \mathrm{H}\right), 7.78\left(\mathrm{~m}, \mathrm{H}_{5}, \mathrm{H}_{6}, 2 \mathrm{H},\right)$, 7.70 (d, Ph, J = 7.50 Hz, 1H), 7.69 (d, H , $\left.^{\prime} \mathrm{J}=8.4 \mathrm{~Hz}, 1 \mathrm{H}\right), 7.50(\mathrm{~m}, \mathrm{Ph}, 2 \mathrm{H}), 7.07$ (d, Ph', 1H), 6.72 (dd, $\left.\mathrm{H}_{6^{\prime}}, \mathrm{J}=7.8 \mathrm{~Hz}, \mathrm{~J}=5.4 \mathrm{~Hz}, 1 \mathrm{H}\right), 6.66\left(\mathrm{~d}, \mathrm{Ph}^{\prime}, \mathrm{J}=8.0 \mathrm{~Hz}, 1 \mathrm{H}\right.$ ), 6.60 (d, Ph', J = 8.0 Hz), 2.52 (s, Me), 2,25 (s, Me). Yield 69\%. MP $341^{\circ} \mathrm{C}(\mathrm{d})$. IR $\left(\mathrm{KBr}, \mathrm{cm}^{-1}\right)$ M-CO 2055.0, 1988.9. Anal.Calcd. (\%) for $\mathrm{C}_{32} \mathrm{H}_{24} \mathrm{Cl}_{2} \mathrm{~N}_{4} \mathrm{O}_{2} \mathrm{Ru}: \mathrm{C}$, 57.49; H, 3.62; N, 8.38. Found (\%): C, 57.20; H, 4.00; N, 8.28.

\section{Cis-[RuCl${ }_{2}(\mathrm{CO})_{2}$ (2-(4'-metoxyphenyl)-1,8-naphthyridine-kN8) ${ }_{2}$ (Ru-5).}

${ }^{1} \mathrm{H}-\mathrm{NMR}\left(200 \mathrm{MHz}_{\mathrm{CDCl}}\right.$ ) 10.54 (dd, $\mathrm{H}_{7}, \mathrm{~J}=5.5 \mathrm{~Hz}, \mathrm{~J}=2.2 \mathrm{~Hz}, 1 \mathrm{H}$ ), 9.03 (dd, $\mathrm{H}_{7}$, $\mathrm{J}=5.7 \mathrm{~Hz}, \mathrm{~J}=1.8 \mathrm{~Hz}, 1 \mathrm{H}), 8.93\left(\mathrm{~m}, \mathrm{H}_{5}, \mathrm{Ph}, 2 \mathrm{H}\right), 8.71\left(\mathrm{~d}, \mathrm{H}_{4}, \mathrm{~J}=8.8 \mathrm{~Hz}, 1 \mathrm{H}\right), 8.68$ $\left(\mathrm{d}, \mathrm{H}_{4}, \mathrm{~J}=8.6 \mathrm{~Hz}, 1 \mathrm{H}\right), 8.59\left(\mathrm{~d}, \mathrm{H}_{3^{\prime}}, \mathrm{J}=8.6 \mathrm{~Hz}, 1 \mathrm{H}\right), 8.42\left(\mathrm{dd}, \mathrm{H}_{5^{\prime}}, \mathrm{J}=8.1 \mathrm{~Hz}, \mathrm{~J}=\right.$ $1.8 \mathrm{~Hz}, 1 \mathrm{H}), 8.21\left(\mathrm{~d}, \mathrm{H}_{3}, \mathrm{~J}=8.8 \mathrm{~Hz}\right), 8.1\left(\mathrm{dd}, \mathrm{H}_{6}, \mathrm{~J}=8.2 \mathrm{~Hz}, \mathrm{~J}=5.5 \mathrm{~Hz}, 1 \mathrm{H}\right), 7.50$ (m, Ph',2H), 7.21 (dd, $\left.\mathrm{H}_{6}, \mathrm{~J}=8.1 \mathrm{~Hz}, \mathrm{~J}=5.7 \mathrm{~Hz}, 1 \mathrm{H}\right), 6.58\left(\mathrm{~m}, \mathrm{Ph}^{\prime}, 2 \mathrm{H}\right), 4.14$ (OMe), 3.91 (OMe). Yield 68\%. MP $337{ }^{\circ} \mathrm{C}(\mathrm{d}) . \mathrm{IR}\left(\mathrm{KBr}, \mathrm{cm}^{-1}\right) \mathrm{M}-\mathrm{CO} 2050.0$, 1988.2. Anal. Calcd. (\%) for $\mathrm{C}_{32} \mathrm{H}_{24} \mathrm{Cl}_{2} \mathrm{~N}_{4} \mathrm{O}_{4} \mathrm{Ru}$ : C, 54.86; $\mathrm{H}, 3.45 ; \mathrm{N}, 8.00$. Found (\%): C, 54.09; H, 3.95; N, 7.98. 


\section{Cis-[RuCl${ }_{2}(\mathrm{CO})_{2}$ (2-(3'-methoxyphenyl)-1,8-naphthyridine-kN8) $\left.{ }_{2}\right](\mathrm{Ru}-6)$.}

${ }^{1} \mathrm{H}-\mathrm{NMR}\left(200 \mathrm{MHz}, \mathrm{CDCl}_{3}\right) 10.47$ (dd, $\mathrm{H}_{7}, \mathrm{~J}=5.4 \mathrm{~Hz}, \mathrm{~J}=1.8 \mathrm{~Hz}, 1 \mathrm{H}$ ), 8.80 (dd, $\left.\mathrm{H}_{7}, \mathrm{~J}=5.3 \mathrm{~Hz}, \mathrm{~J}=1,8 \mathrm{~Hz}, 1 \mathrm{H}\right), 8.41\left(\mathrm{dd}, \mathrm{H}_{5}, \mathrm{~J}=7.9 \mathrm{~Hz}, \mathrm{~J}=1.8 \mathrm{~Hz}, 1 \mathrm{H}\right), 8.23(\mathrm{~m}$, $\mathrm{Ph}, 2 \mathrm{H}), 7.98\left(\mathrm{~m}, \mathrm{H}_{4^{\prime}}, \mathrm{H}_{4}, 2 \mathrm{H}\right), 7.79\left(\mathrm{~m}, \mathrm{H}_{3^{\prime}}, \mathrm{H}_{5^{\prime}}, \mathrm{H}_{3}, 3 \mathrm{H}\right), 7.58\left(\mathrm{~m}, \mathrm{H}_{6}, \mathrm{Ph}, 2 \mathrm{H}\right), 7.18$ $(\mathrm{m}, \mathrm{Ph}, 1 \mathrm{H}), 6.91\left(\mathrm{~m}, \mathrm{Ph}^{\prime}, 1 \mathrm{H}\right), 6.68\left(\mathrm{~m}, \mathrm{H}_{6}, \mathrm{Ph}^{\prime}, \mathrm{Ph}, 4 \mathrm{H}\right), 4.05$ (s, m-OMe), 3.35 (s, $\mathrm{m}-\mathrm{OMe})$. Yield $62 \%$. MP $358{ }^{\circ} \mathrm{C}(\mathrm{d})$. IR $\left(\mathrm{KBr}, \mathrm{cm}^{-1}\right) \mathrm{M}-\mathrm{CO} 2055.5$, 1986. Anal. Calcd. (\%) for $\mathrm{C}_{32} \mathrm{H}_{24} \mathrm{Cl}_{2} \mathrm{~N}_{4} \mathrm{O}_{4} \mathrm{Ru}$ : C, 54.86; $\mathrm{H}, 3.45 ; \mathrm{N}, 8.00$. Found (\%): C, 54.06; $\mathrm{H}, 3.53 ; \mathrm{N}, 8.10$.

\section{Cis-[RuCl${ }_{2}(\mathrm{CO})_{2}$ (2-(2'-methoxiphenyl)-1,8-naphthyridine-kN8) $\left.{ }_{2}\right](\mathrm{Ru}-7)$.}

${ }^{1} \mathrm{H}-\mathrm{NMR}\left(200 \mathrm{MHz}, \mathrm{CDCl}_{3}\right) 10.54$ (dd, $\mathrm{H}_{7}, \mathrm{~J}=5.5 \mathrm{~Hz}, \mathrm{~J}=2.0 \mathrm{~Hz}, 1 \mathrm{H}$ ), 9.03 (dd, $\left.\mathrm{H}_{7^{\prime}}, \mathrm{J}=5.7 \mathrm{~Hz}, \mathrm{~J}=1,8 \mathrm{~Hz}, 1 \mathrm{H}\right), 8.93\left(\mathrm{~m}, \mathrm{H}_{5}, \mathrm{Ph}, 3 \mathrm{H}\right), 8.68\left(\mathrm{~d}, \mathrm{H}_{4}, \mathrm{~J}=8.6 \mathrm{~Hz}, 1 \mathrm{H}\right)$, $8.71(\mathrm{~d}, \mathrm{H} 4, \mathrm{~J}=8.8 \mathrm{~Hz}, 1 \mathrm{H}), 8.59\left(\mathrm{~d}, \mathrm{H}_{3^{\prime}}, \mathrm{J}=8.6 \mathrm{~Hz}, 1 \mathrm{H}\right), 8.42\left(\mathrm{dd}, \mathrm{H}_{5^{\prime}}, \mathrm{J}=8.1 \mathrm{~Hz}\right.$, $\mathrm{J}=1.8 \mathrm{~Hz}, 1 \mathrm{H}), 8.21\left(\mathrm{~d}, \mathrm{H}_{3}, \mathrm{~J}=8.8 \mathrm{~Hz}, 1 \mathrm{H}\right), 8.10\left(\mathrm{dd}, \mathrm{H}_{6}, \mathrm{~J}=8.2 \mathrm{~Hz}, \mathrm{~J}=5.5 \mathrm{~Hz}\right.$, $1 \mathrm{H}), 7.50\left(\mathrm{~m}, \mathrm{Ph}, \mathrm{Ph}^{\prime}, 4 \mathrm{H}\right), 7.21\left(\mathrm{~d}, \mathrm{H}_{6^{\prime}}, 1 \mathrm{H}, \mathrm{J}=8.1 \mathrm{~Hz}, \mathrm{~J}=5.71 \mathrm{~Hz}, 1 \mathrm{H}\right), 6.58(\mathrm{~m}$, $\mathrm{Ph}^{\prime} .2 \mathrm{H}$ ), 4.14 (s, o-OMe), 3.91 (s, o-OMe). Yield 50\%. MP $287^{\circ} \mathrm{C}$ (d). IR (KBr, $\mathrm{cm}^{-1}$ ) M-CO; 2056.0, 1989.0. Anal. Calcd. (\%) for $\mathrm{C}_{32} \mathrm{H}_{24} \mathrm{Cl}_{2} \mathrm{~N}_{4} \mathrm{O}_{4} \mathrm{Ru}$ : C, 54.86; $\mathrm{H}$, $3.45 ; \mathrm{N}, 8.00$. Found (\%): C, 54.04; H, 3.91; N, 7.89 .

\section{Catalytic procedure.}

In a glass reactor fitted with a condenser, $\mathrm{NaOH}(0.475 \mathrm{mmol})$ was added to propan-2-ol $(5 \mathrm{~mL})$ and stirred for 1 hour at $80{ }^{\circ} \mathrm{C}$ under nitrogen. Next, the ruthenium complex $(0.0125 \mathrm{mmol})$, propan-2-ol $(4 \mathrm{~mL})$ and a solution $(1 \mathrm{~mL})$ of $p$ cymene as internal standard $(1.92 \mathrm{mmol})$ were added to the corresponding substrate $(2.56 \mathrm{mmol})$ dissolved in propan-2-ol completing a total volume of $10 \mathrm{~mL}$. Identities of reaction components were determined by comparison with commercial samples by gas chromatography.

\section{Results and discussion.}

A series of ruthenium (II) complexes with different substituted naphthyridine ligands have been prepared and characterized. The ligands L1-L7 were synthesized from 2-aminonicotinaldehyde and the corresponding substituted acetophenone (Scheme 2). 
<smiles>[R]c1c(C(C)=O)ccc(-c2ccc(-c3ccc4cccnc4n3)c([R])c2[R])c1[R]</smiles>

$\mathbf{L}_{1}: \mathbf{R}_{1}=\mathbf{H}, \mathbf{R}_{2}=\mathrm{H}, \mathbf{R}_{3}=\mathrm{H} ; \mathbf{L}_{2}: \mathbf{R}_{1}=\mathrm{NO}_{2}, \mathbf{R}_{2}=\mathrm{H}, \mathbf{R}_{3}=\mathrm{H} ; \mathbf{L}_{3}: \mathbf{R}_{1}=\mathrm{Br}, \mathbf{R}_{2}=\mathrm{H}, \mathbf{R}_{\mathbf{3}}=\mathrm{H}$

$\mathbf{L}_{4}: \mathbf{R}_{1}=\mathrm{CH}_{3}, \mathbf{R}_{2}=\mathrm{H}, \mathbf{R}_{3}=\mathrm{H} ; \mathbf{L}_{5}: \mathbf{R}_{1}=O \mathrm{OCH}_{3}, \mathbf{R}_{2}=\mathrm{H}, \mathbf{R}_{3}=\mathrm{H} ; \mathbf{L}_{6}: \mathbf{R}_{1}=\mathrm{H}, \mathbf{R}_{2}=\mathrm{OCH}_{3}, \mathbf{R}_{3}=\mathrm{H}$

$$
\mathbf{L}_{7}: \mathbf{R}_{1}=\mathbf{H}, \mathbf{R}_{2}=\mathbf{H}, \mathbf{R}_{3}=\mathrm{OCH}_{3}
$$

Scheme 2. Synthesis of 1,8-naphthyridine ligands (1,8 NAPs).

Then, the $\mathbf{R u}(\mathrm{II})$ complexes $\mathbf{R u} \mathbf{- 1}-\mathbf{R u} \mathbf{- 7}$ were readily prepared by reaction of the carbonyl precursor $\left[\mathrm{RuCl}_{2}(\mathrm{CO})_{2}\right]_{2}$ and the naphthyridine derivatives $\mathrm{L}$ by the procedures reported in the literature [56-60].
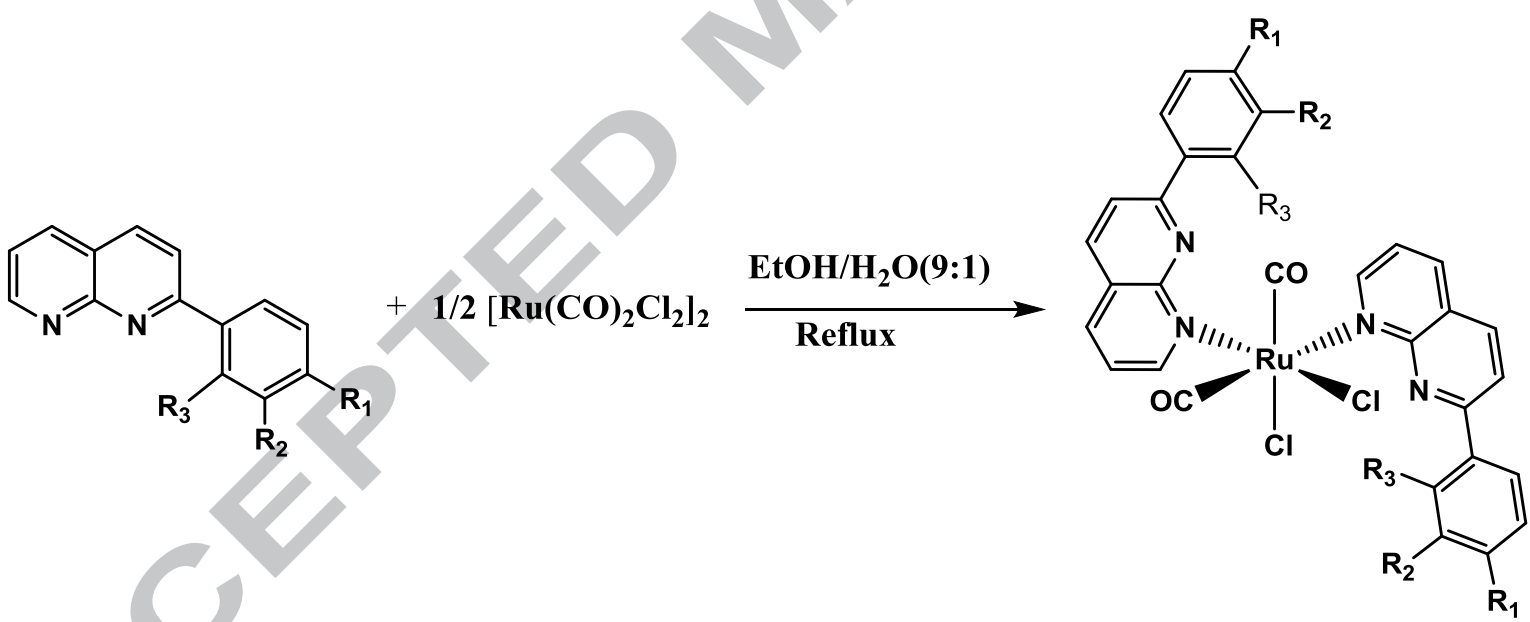

Ru-1: $\mathbf{R}_{1}=H, \mathbf{R}_{2}=H, \mathbf{R}_{3}=H$; Ru-2: $\mathbf{R}_{1}=\mathrm{NO}_{2}, \mathbf{R}_{2}=\mathrm{H}, \mathbf{R}_{3}=\mathrm{H} ; \mathrm{Ru}-3: \mathbf{R}_{1}=\mathrm{Br}, \mathbf{R}_{2}=\mathrm{H}, \mathbf{R}_{\mathbf{3}}=\mathrm{H}$

Ru-4: $\mathbf{R}_{1}=\mathrm{CH}_{3}, \mathbf{R}_{2}=\mathrm{H}, \mathbf{R}_{3}=\mathrm{H} ; \mathrm{Ru}-5: \mathrm{R}_{1}=\mathrm{OCH}_{3}, \mathrm{R}_{2}=\mathrm{H}, \mathrm{R}_{3}=\mathrm{H} ; \mathrm{Ru}-6: \mathbf{R}_{1}=\mathrm{H}, \mathbf{R}_{2}=\mathrm{OCH}_{3}, \mathbf{R}_{3}=\mathrm{H}$

$$
\text { Ru-7: } \mathbf{R}_{\mathbf{1}}=\mathrm{H}, \mathbf{R}_{\mathbf{2}}=\mathrm{H}, \mathbf{R}_{\mathbf{3}}=\mathrm{OCH}_{3}
$$

Scheme 3. Synthesis of ruthenium complexes containing naphthyridine ligands.

All the complexes $\mathrm{RuCl}_{2}(\mathrm{CO})_{2}(\mathbf{L})_{2}$ were isolated in good yield. Two 1,8naphthyridine ligands $\mathbf{L}$ coordinate to the metal center in a $\mathrm{N}$-monodentate fashion. Whatever the reaction conditions, we do not observe the formation complexes 
containing $\mathrm{N}, \mathrm{N}$-bidentate $\mathrm{L}$ ligands. Examples of $\mathrm{N}, \mathrm{N}$-bidentate coordination of naphthyridines have been reported in the case of the phosphine complex $\mathrm{Ru}(\mathbf{L})\left(\mathrm{PPh}_{3}\right)_{2} \mathrm{Cl}_{2}[57]$.

The chemical structure of the complexes, Ru-5, and Ru-7 were confirmed by XRay crystallographic studies (Figures 1 and 2). Table 1 presents selected bond lengths and Table 2 selected bond angles. The two complexes have an octahedral geometry, in which the two naphthyridine ligands are cis to each other, one is trans to a chloride and the second one is trans to a carbonyl. The high sterical hindrance of phenyl substituted naphthyridine ligands does not preclude a cis configuration.

The bond distances and bond angles are similar to those found in related complexes reported by us [53]. 


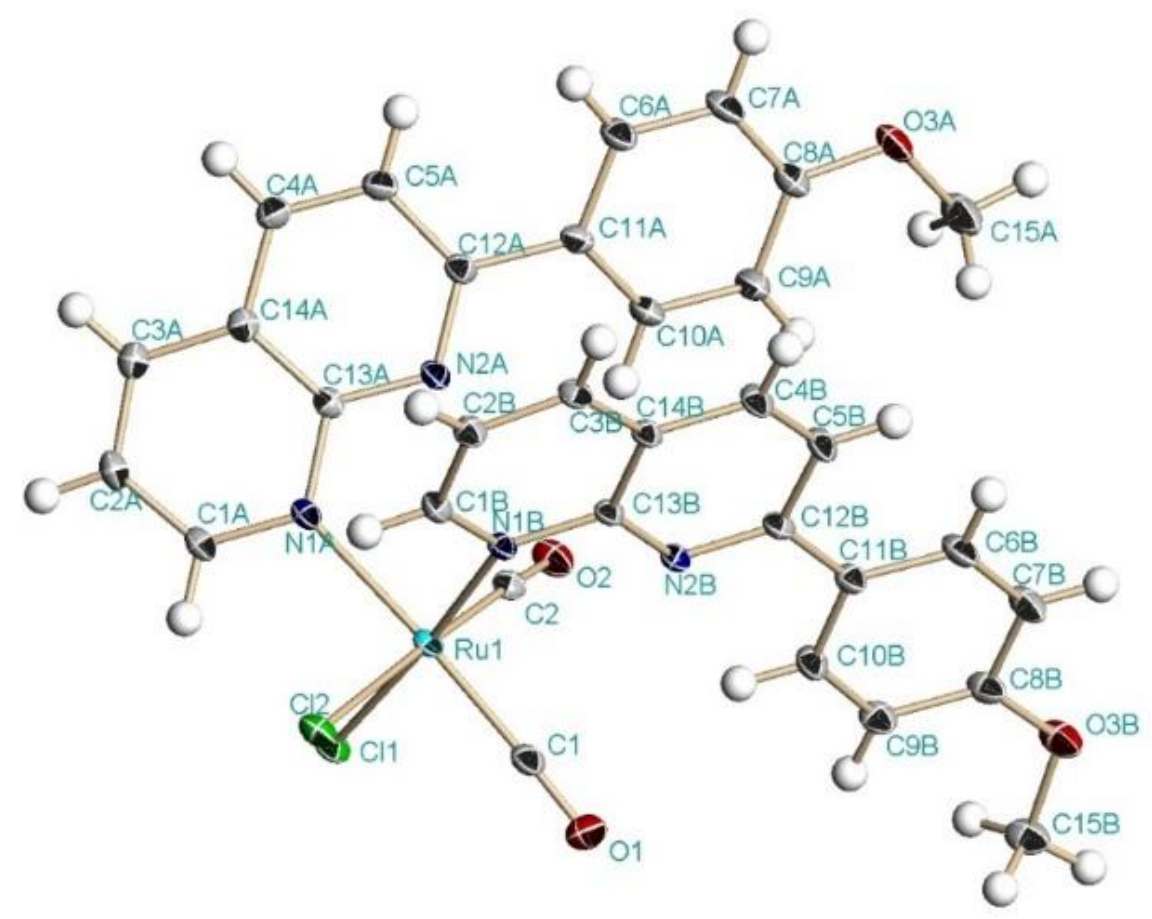

Figure 1. Molecular view of the complex cis-[RuCl${ }_{2}(\mathrm{CO})_{2}\left(2-\left(4^{\prime}-\right.\right.$ methoxyphenyl)1,8-naphthyridine-kN8) 2 , Ru-5 showing the labeling scheme. 


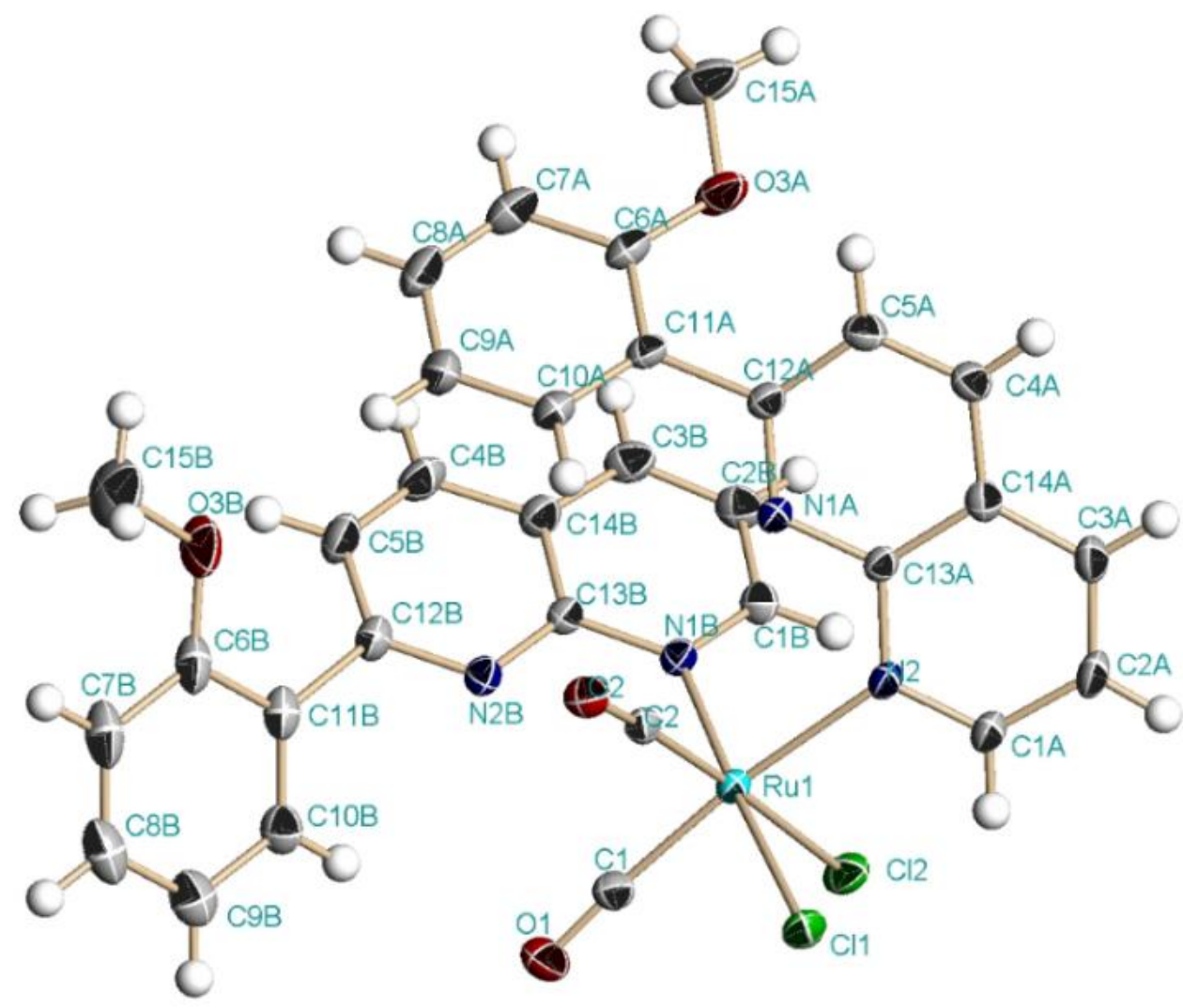

Figure 2. Molecular view of the complex cis- $\left[\operatorname{RuCl}_{2}(\mathrm{CO})_{2}(2-(2\right.$ '-methoxyphenyl)1,8-naphthyridine-kN8) ${ }_{2}$, (Ru-7) showing the labeling scheme. 


\section{Catalytic results.}

Table 3 summarizes the catalytic activity in the transfer hydrogenation of acetophenone shown by the ruthenium (II) complexes Ru-1 to Ru-5 in the presence of $\mathrm{NaOH}$. In all cases, only one product, 1-phenyl ethanol, is obtained with $100 \%$ selectivity. Entry 6 shows that the best activity $(66 \%$ and $70 \%$ of conversion) after 1 hour of reaction is observed for Ru-4 and Ru-5 respectively. The lesser is found for $\mathbf{R u - 2}\left(\mathrm{R}_{1}=\mathrm{NO}_{2}\right)$. In the hydrogen transfer reaction, the first ten minutes of reaction is very important since the reaction of the precatalyst with propan-2-ol allows the formation of the actives species of the reaction, during this time the metal hydride is formed. The proposed mechanism of the hydrogen transfer reaction suggests that the ruthenium hydride is the active species in this reaction (Figure 3).

The results reported here (table 3) show that the formation of the active species (the dihydride complex) (see (Figure 3) could depend on the nature of the ligand $\mathrm{L}$. The presence of the electron-withdrawing group into the ligand $\left(\mathrm{L}_{3}\right)\left(\mathrm{NO}_{2}\right.$ and $\left.\mathrm{Br}\right)$ could be disfavor the dihydride complex formation showing a lower conversion during the first thirty minutes of reaction. When the ruthenium hydride active species is formed the conversion for all catalysts become similar (Entry 8), where all catalysts converge to a maximum conversion between 80 to $95 \%$. With the aim of optimizing the catalyst activity of Ru-4 that showed the larger activity, we did comparative studies using two types of base: $\mathrm{NaOH}$ and t-BuOK. The role of the base is essential as it activates the coordinated isopropanoxy leading to the formation of the hydride species (Figure 3). The results are summarized in Table 4. During the course of the reaction, the TOF fluctuates, due to the decrease in substrate concentration during the catalytic reaction, affecting the conversion rate. 
Table 3. Catalytic activities in the hydrogen transfer reaction of acetophenone with ruthenium complexes containing naphthyridine ligands.

\begin{tabular}{|c|c|c|c|c|c|c|}
\hline & \multicolumn{7}{|c|}{ Conversion (\%) Complexes } \\
\hline Run & Time(min) & Ru-1 & Ru-2 & Ru-3 & Ru-4 & Ru-5 \\
\hline 1 & 5 & 32 & 23 & 28 & 36 & 39 \\
\hline 2 & 10 & 35 & 23 & 29 & 38 & 41 \\
\hline 3 & 15 & 35 & 29 & 33 & 40 & 45 \\
\hline 4 & 30 & 42 & 31 & 36 & 56 & 53 \\
\hline 5 & 45 & 53 & 35 & 45 & 63 & 67 \\
\hline 6 & 60 & 57 & 35 & 53 & 66 & 70 \\
\hline 7 & 185 & 78 & 74 & 79 & 84 & 79 \\
\hline 8 & 300 & 87 & 85 & 88 & 91 & 95 \\
\hline
\end{tabular}

Reaction conditions: acetophenone $(2.56 \mathrm{mmol})$, catalyst $(0.0125 \mathrm{mmol}), \mathrm{NaOH}(0.475 \mathrm{mmol}), \mathrm{p}$-cymene $(1.92 \mathrm{mmol})$ propan-2-ol $(10 \mathrm{~mL}), 8{ }^{\circ} \mathrm{C}, \mathrm{N}_{2}$. Substrate/catalyst ratio $=205 / 1$. cis- $\left[\mathrm{RuCl}_{2} \mathrm{CO}\right)_{2}\left(2\right.$-phenyl-1,8-naphthyridine-kN8) $\left.{ }_{2}\right](\mathbf{R u}-1)$, cis- $\left[\mathrm{RuCl}_{2}(\mathrm{CO})_{2}\left(2-\left(4^{\prime} \text {-nitrophenyl)-1,8-naphthyridine-kN8 }\right)_{2}\right] \quad(\mathbf{R u}-2)\right.$, cis-[RuCl${ }_{2}(\mathrm{CO})_{2}(2-(4$ '-bromophenyl)-1,8- naphthyridine$\left.\left.\mathrm{KN} 8)_{2}\right](\mathbf{R u}-3),\right)$, cis-[RuCl${ }_{2}(\mathrm{CO})_{2}\left(2-\left(4^{\prime}-\text {-methylphenyl)-1,8-naphthyridine-kN8 }\right)_{2}\right](\mathbf{R u}-4), \quad c i s-\left[\mathrm{RuCl}_{2}(\mathrm{CO})_{2}\left(2-\left(4^{\prime}-\right.\right.\right.$ methoxyphenyl)1,8-naphthyridine-kN8) 2 ] (Ru-5).

Using the inorganic base $(\mathrm{NaOH})$ as a co-catalyst and after 1 hour of reaction, the activity of catalyst $\mathbf{R u}-\mathbf{4}$ is $66 \%$ and the turnover frequency (TOF) is $135 \mathrm{~h}^{-1}$, this activity is similar for catalyst Ru-5. This latter value is greater that than obtained by employing of t-BuOK. Our group have been studied the hydrogen transfer using different ruthenium complexes containing polypyridine ligands which showed that the complex containing polypiridine ligands are active catalysts in this reaction $[53,55]$. The catalyst loading was also studied with the goal of optimizing the maximum substrate concentration at which the catalyst shows good performance. 
Table 4. Effect of the base in the transfer hydrogenation of acetophenone using the complex cis-[RuCl $2(\mathrm{CO})_{2}\left(2-(4 \text { '-methylphenyl)-1,8-naphthyridine-kN8 })_{2}\right],(\mathbf{R u}-4)$ as precatalyst.

\begin{tabular}{|c|c|c|c|c|}
\hline & \multicolumn{2}{|c|}{ Base NaOH } & \multicolumn{2}{c|}{ Base t-BuOK } \\
\hline Time (min) & $\begin{array}{c}\text { Conversion } \\
(\%)\end{array}$ & TOF $\left(\mathbf{h}^{-1}\right)$ & $\begin{array}{c}\text { Conversion } \\
\%\end{array}$ & TOF $\left(\mathbf{h}^{-1}\right)$ \\
\hline 5 & 36 & 886 & 44 & 1082 \\
\hline 10 & 38 & 467 & 44 & 542 \\
\hline 15 & 40 & 328 & 44 & 361 \\
\hline 30 & 56 & 230 & 44 & 180 \\
\hline 45 & 63 & 172 & 50 & 137 \\
\hline 60 & 66 & 135 & 50 & 103 \\
\hline
\end{tabular}

Reaction conditions: acetophenone $(2.56 \mathrm{mmol})$, catalyst $(0.0125 \mathrm{mmol})$, base $(0.475 \mathrm{mmol}), \mathrm{p}$ cymene $(1.92 \mathrm{mmol})$, propan-2-ol $(10 \mathrm{~mL}), 80^{\circ} \mathrm{C}, \mathrm{N}_{2}$. Substrate/catalyst ratio $=205 / 1$.

Table 5 shows the conversion and the turnover frequencies obtained using different substrate/catalyst ratio for 1 hour of reaction. If the substrate/catalyst ratio is larger, the TOF value increases. Thus, in the case of a ratio of $1000 / 1$, the TOF value is $390 \mathrm{~h}^{-1}$. We consider that this ratio is optimal, although the conversion, down to $39 \%$, is only $3 \%$ lesser than that obtained when the substrate catalyst ratio is $700 / 1$. Although the conversion diminishes as the concentration of catalyst increases, the turnover frequency is higher. By contrast, when the reaction is performed between 3-5 hours, the conversions were close to $95 \%$ whatever the substrate/catalyst ratio used. The mechanism of hydrogen transfer reaction has been reported by several authors. Van Leeuwen [61,62] reported a detailed mechanism for this reaction. In a first stage, the dichloro precursor is converted into the metal dihydride complex (b) following the reported mechanism. 
Table 5. Effect of the substrate/catalyst ratio in the transfer hydrogenation of acetophenone with the complex cis-[RuCl $2(\mathrm{CO})_{2}(2-(4$ '-methylphenyl)-1,8naphthyridine-kN8) 2], (Ru-4).

\begin{tabular}{|c|c|c|}
\hline Substrate/catalyst & $\begin{array}{c}\text { Conversion } \\
\%\end{array}$ & $\mathbf{T O F}\left(\mathbf{h}^{-1}\right)$ \\
\hline $205 / 1$ & 66 & 135 \\
\hline $500 / 1$ & 47 & 235 \\
\hline $700 / 1$ & 42 & 294 \\
\hline $1000 / 1$ & 39 & 390 \\
\hline
\end{tabular}

Reaction conditions: substrate acetophenone, catalyst $(0.0125 \mathrm{mmol}), \mathrm{NaOH}(0.475 \mathrm{mmol}), \mathrm{p}$ cymene (1.92 mmol), propan-2-ol (10 mL), $80{ }^{\circ} \mathrm{C}, \mathrm{N}_{2}$. Reaction time:1 hour.

Then addition of the ketone gives rises to the formation of the [Ru]$\mathrm{OC}\left(\mathrm{CH}_{3}\right)(\mathrm{H})(\mathrm{Ph})(\mathbf{c})$ species. The species (c) undergoes reductive elimination to form a tetracoordinate metal complex (d) together with a molecule of the hydrogenated product which leaves the cycle. Then this tetracoordinate intermediate reacts with propanol-2-ol to generate the metal hydride complex (e). Finally, species (e) through a ß-elimination produces acetone regenerating the dihydride hexacoordinate complex (b), thus closing the catalytic cycle. 

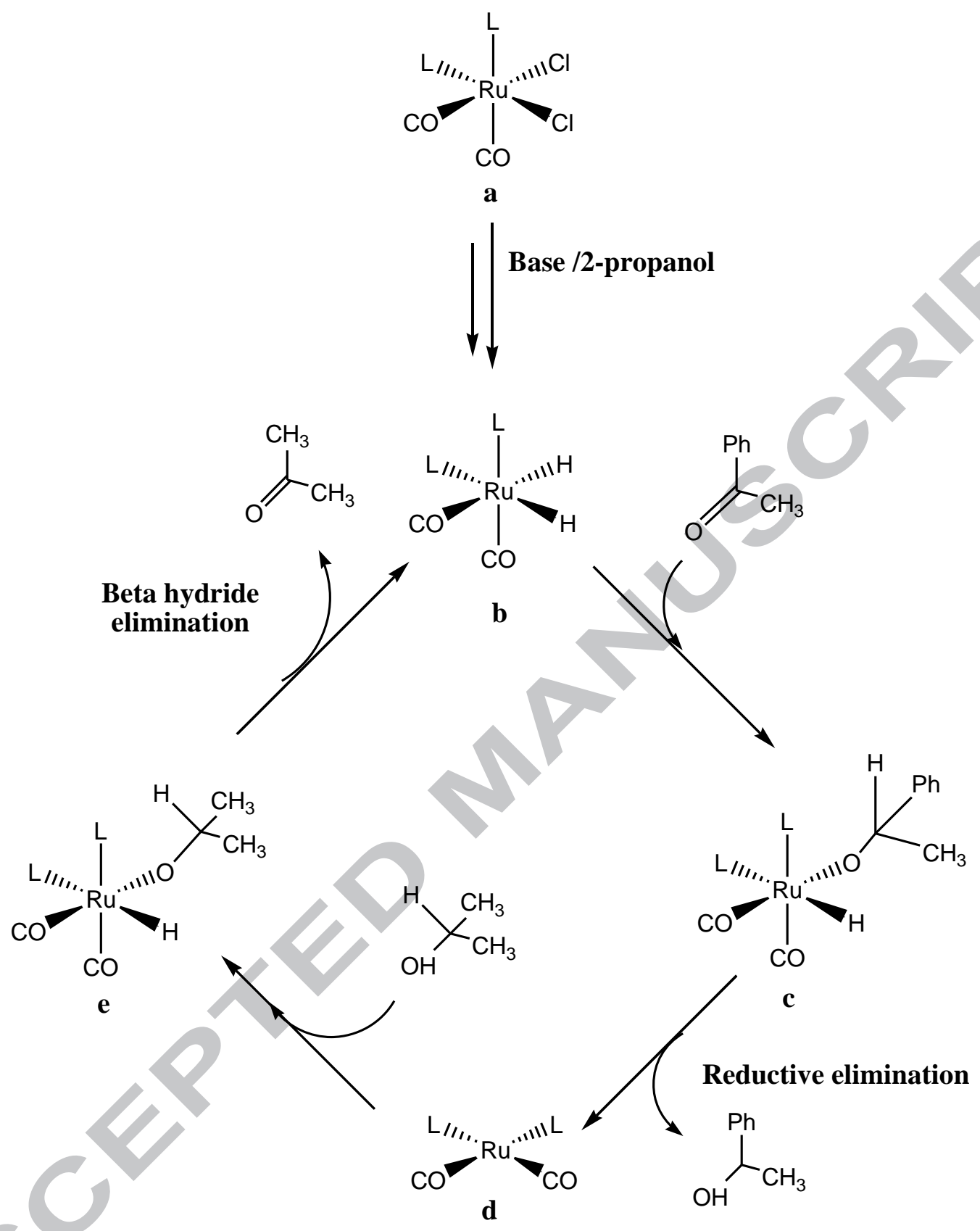

\section{$\mathrm{L}=$ 1,8-Napthyridine ligands}

Figure 3. Proposed mechanism for the hydrogen transfer reaction of acetophenone. 
Correlation between Hammet parameters for the substrate substituent and conversion, is shown in Table 6, when the catalyst $\mathbf{R u - 4}$ was studied in the hydrogen transfer reaction. When a chloride is incorporated in the para-position of the phenyl group, the conversion improves by $20 \%$ with respect to the unsubstituted substrate. By contarst, when an electron-donating group, a $p$-methyl or $p$-methoxy is introduced into the substrate, the activity decreases considerably. Based on the mechanism proposed for this reaction, the presence of an electronwithdrawing on the substrate favors hydride attack (step (c) $\rightarrow$ (d), (Figure 3 ). The hydrogen transfer to the substrate is favored by the presence of the chloride substituent on the phenyl ring, as illustrated by Figure 4 which shows good correlation between Hammet parameters and TOF values. This suggests that the step $(\mathbf{c}) \rightarrow(\mathbf{d})$, is the determining step in the transfer hydrogenation reaction of acetophenone by ruthenium(II) complexes incorporating naphthyridine ligands. Additionally, the study of the activity vs. time (Table 3) confirms that the formation of a metal hydride (b) depends on the nature of the naphthyridine ligand.

Table 6. Effect of the substrate in hydrogen transfer reaction of acetophenone using the cis-[RuCl $2\left(2-(4 \text { '-methylphenyl)-1,8-naphthyridine-kN8 })_{2}(\mathrm{CO})_{2}\right]$ complex, Ru-5.

\begin{tabular}{|c|c|c|}
\hline Substrate & $\begin{array}{c}\text { Conversion } \\
\%\end{array}$ & Hammett parameter \\
\hline$p-\mathrm{ClC}_{6} \mathrm{H}_{4}-\mathrm{C}(\mathrm{O})-\mathrm{CH}_{3}$ & 77 & 0,23 \\
\hline $\mathrm{C}_{6} \mathrm{H}_{4} \mathrm{C}(\mathrm{O})-\mathrm{CH}_{3}$ & 57 & 0,0 \\
\hline$p-\mathrm{CH}_{3}-\mathrm{C}_{6} \mathrm{H}_{4}-\mathrm{C}(\mathrm{O})-\mathrm{CH}_{3}$ & 35 & $-0,17$ \\
\hline$p-\mathrm{CH}_{3} \mathrm{O}-\mathrm{C}_{6} \mathrm{H}_{4}-\mathrm{C}(\mathrm{O})-\mathrm{CH}_{3}$ & 23 & $-0,27$ \\
\hline
\end{tabular}

Reaction conditions: substrate $(2.56 \mathrm{mmol})$, pre-catalyst $(0.0125 \mathrm{mmol}), \mathrm{NaOH}(4.75 \mathrm{mmol}), \mathrm{p}-$ cymene $(1.92 \mathrm{mmol})$, propanol-2-ol $(10 \mathrm{~mL}), 80^{\circ} \mathrm{C}, \mathrm{N}_{2}$. Substrate $/$ catalyst ratio $=205 / 1$ 


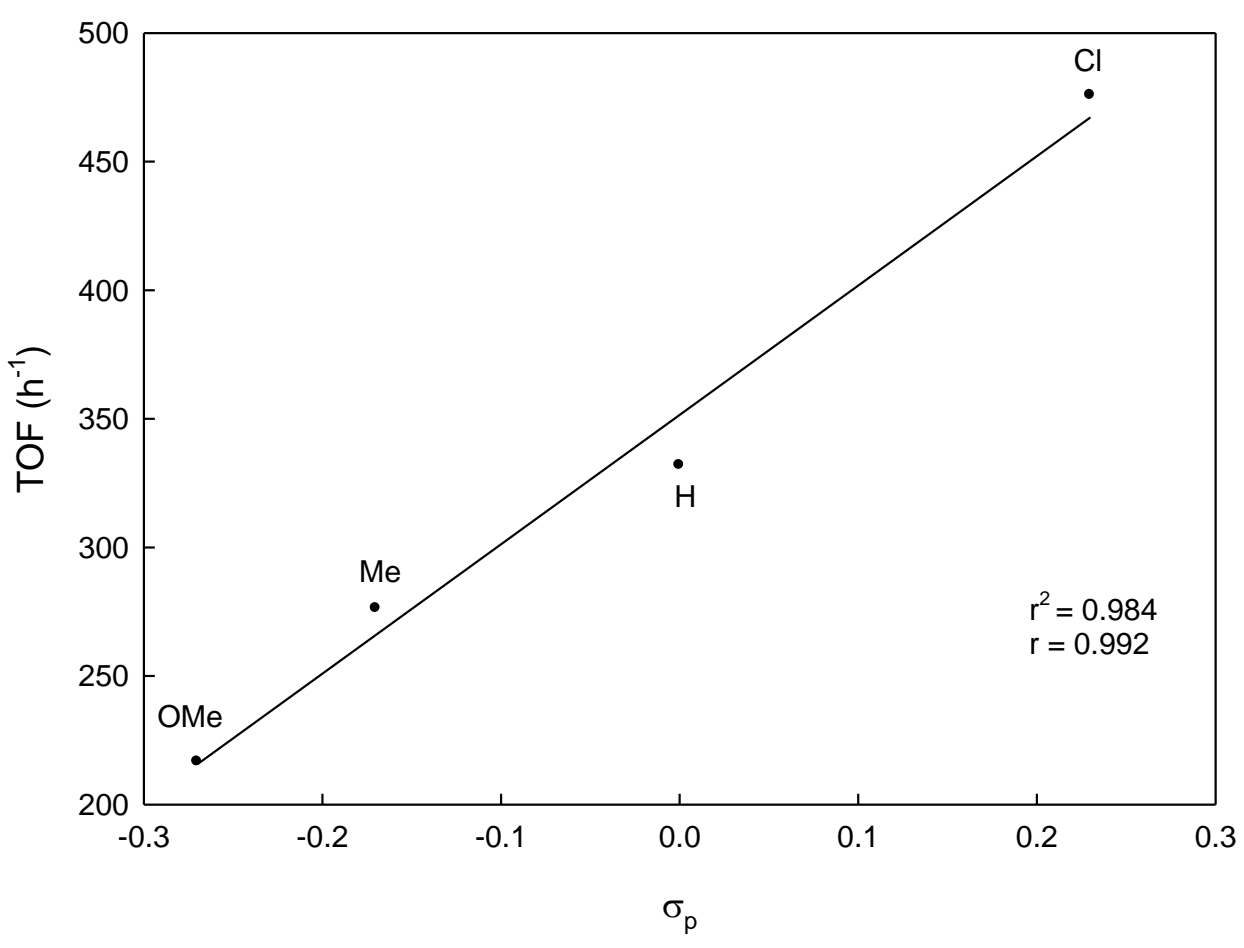

Figure 4. Correlation between Hammett parameter and the turnover frequency for different substrates in hydrogen transfer reaction of Ru-5.

\section{Conclusions}

A series of ruthenium(II) complexes of the general molecular formula $R u$ $(\mathrm{CO}) \mathrm{Cl}_{2}(\mathbf{L})_{2}$ (where $\mathbf{L}=$ substituted naphthyridine ligands) were successfully synthesized and characterized. The characterization of the complexes was accomplished by standard analytical and spectral methods. Single crystal X-ray diffraction analysis confirms the coordination of two naphthyridine ligands to the metal, showing that they are bonded to the metal through a monodentate $N$ coordination mode, generating a distorted octahedral structure.

We have demonstrated that all complexes exhibit a moderate catalytic activity in the transfer hydrogenation of acetophenone in the presence of a base. It is noteworthy that these complexes exhibit $100 \%$ selectivity, the corresponding 
alcohol being formed and a low load of the base $(\mathrm{NaOH})$ is required in order to achieve the observed activities.

The catalyst (Ru-5) shown turnover frequency value between 135-390 $\mathrm{h}^{-1}$, depending the substrate/catalyst ratio. In addition, the catalyst is active for various acetophenone derivatives. The reaction is sensitive to the nature of the substituent on the phenyl ring. The best activity is obtained when the ring was substituted by $\mathrm{Cl}$ in para position, indicating that the rate-determining step is the reductive elimination.

\section{Acknowledgments}

This work has been supported by ECOS-CONICYT (Action C14E02) and Fondecyt 1160505. J. C. Araya and J. Gajardo gratefully acknowledge CONICYT-Chile for their doctoral fellowships. S.A.M acknowledges the financial support provided by DICYT-USACH-Chile. This research has been performed as part of the ChileanFrench “Joint Laboratory for Inorganic Functional Materials” (LIA MIF). 


\section{References}

[1] M.A. Ciriano, L.A. Oro, Comprehensive Coordination Chemistry II, vol. 1, Elsevier, Oxford, 2004, pp. 55.

[2] S.P. Stanforth, Comprehensive Heterocyclic Chemistry II, vol. 7, Elsevier, Oxford, 1996, pp. 527.

[3] P. Caluwe, G. Evens, Macromolecules 12 (1979) 803-808.

[4] R.P. Thummel, F.D. Cantu, R.J. Mahadevan, J. Org. Chem. 49 (1984) 2208.

[5] T.G. Majewicz, P. Caluwe, J. Org. Chem. 39 (1974) 720.

[6] E.M. Hawes, D.G. Wibberley, J. Chem. Soc. C (1967) 1564.

[7] a) C.S. Campos-Fernández, L.M. Thomson, J.R. Galán-Mascarós, X. Ouyang, K.R. Dunbar, Inorg. Chem. 41 (2002) 1523. B) N.R. Rivera, Y. Hsiao, J.A. Cowen, C. McWilliams, J. Armstrong, N. Yasuda, D.L. Hughes, Synth. Commun. 31 (2001) 1573.

[8] S. Adhikari, O. Hussain, R.M. Phillips and M.R.Kollipara, J.Organomet.Chem 854 (2018) 27.

[9] M.S. Majumdar, K. Patra, M. Kannan, K.R. Dunbar, J.K. Bera, Inorg. Chem. 47 (2008) 2212.

[10] S.K. Patra, M. Majumdar, J.K. Bera, J. Organomet. Chem. 691 (2006) 4779.

[11] S.K. Patra, N. Sadhukhan, J.K. Bera, Inorg. Chem. 45 (2006) 4007.

[12] S.K. Patra, J.K. Bera, Organometallics 25 (2006) 6054.

[13] P.G. Dormer, K.K. Eng, R.N. Farr, G.R. Humphrey, J.C. McWilliams, P.J.

Reider, J. W. Sager, R.P. Volante, J. Org. Chem. 68 (2003) 467.

[14] Y. Hsiao, N.R. Rivera, N. Yasuda, D.L. Hughes, P.J. Reider, Org. Lett. 3 (2001) 1101.

[15] S.A. Moya, J. Gajardo, J.C. Araya, J.J. Cornejo, V. Guerchais, H. Le Bozec, J.C. Bayón, A.J. Pardey, P. Aguirre, Appl. Organomet. Chem. 22 (2008) 471.

[16] Wei-Hung Tang, Yi-Hung Liu, Shie-Ming Peng, Shiuh-Tzung Liu Journal of Organometallic Chemistry 775 (2015) 94.

[18] E.O. Ozcan, D. Mercan, N. Gurbuz, E. Cetinkaya, B. Cetinkaya, I. Ozdemir, Turk J. Chem. 35 (2011) 699.

S. Adhikari, O.Hussain, R. M. Phillips, M.R. Kollipara . Journal of Organometallic Chemistry 854 (2018) 27.

[19] M.D. Fryzuk, P.A. MacNeil, Organometallics 2 (1983) 682

[20] K. Abdur-Rashid, M. Faatz, A.J. Lough, R.H. Morris, J. Am. Chem. Soc. 123 (2001) 7473.

[21] K. Murata, H. Konishi, M. Ito, T. Ikariya, Organometallics 21 (2002) 253.

[22] P. Maire, T. Büttner, F. Breher, P. Le Floch, H. Grützmacher, Angew. Chem. Int. Ed. 44 (2005) 6318. 
[23] D. Sellmann, R. Prakash, F.W. Heinemann, M. Moll, M. Klimowicz, Angew. Chem. Int. Ed. 43 (2004) 1877.

[24] Y. Ohki, M. Sakamoto, K. Tatsumi, J. Am. Chem. Soc. 130 (2008) 11610. [25] H.F.T. Klare, M. Oestreich, J.-I. Ito, H. Nishiyama, Y. Ohki, K. Tatsumi, J. Am. Chem. Soc. 133 (2011) 3312.

[26] T. Stahl, K. Müther, Y. Ohki, K. Tatsumi, M. Oestreich, J. Am. Chem. Soc. 135 (2013) 10978.

[27] Y. Misumi, H. Seino, Y. Mizobe, J. Am. Chem. Soc. 131 (2009) 14636.

[28] H. Seino, Y. Misumi, Y. Hojo, Y. Mizobe, Dalton Trans. 39 (2010) 3072.

[29] E.J. Derrah, D.A. Pantazis, R. McDonald, L. Rosenberg, Organometallics 26 (2007) 1473.

[30] P.J. Walsh, F.J. Hollander, R.G. Bergman, J. Am. Chem. Soc. 110 (1988) 8729.

[31] C.C. Cummins, S.M. Baxter, P.T. Wolczanski, J. Am. Chem. Soc. 110 (1988) 8731.

[32] C.C. Cummins, C.P. Schaller, G.D. Van Duyne, P.T. Wolczanski, A.W.E. Chan, R. Hoffmann, J. Am. Chem. Soc. 113 (1991) 2985.

[33] T.I. Gountchev, T.D. Tilley, J. Am. Chem. Soc. 119 (1997) 12831.

[34] J.L. Polse, R.A. Andersen, R.G. Bergman, J. Am. Chem. Soc. 120 (1998) 13405.

[35] R.E. Blake Jr., D.M. Antonelli, L.M. Henling, W.P. Schaefer, K.I. Hardcastle, J.E. Bercaw, Organometallics 17 (1998) 718.

[36] D.J.M. Trosch, P.E. Collier, A. Bashall, L.H. Gade, M. McPartlin, P. Mountford, S. Radojevic, Organometallics 20 (2001) 3308.

[37] T.E. Hanna, I. Keresztes, E. Lobkovsky, W.H. Bernskoetter, P.J. Chirik, Organometallics 23 (2004) 3448.

[38] N. Hazari, P. Mountford, Acc. Chem. Res. 38 (2005) 839.

[39] H.S. La Pierre, J. Arnold, F.D. Toste, Angew. Chem. Int. Ed. 50 (2011) 3900.

[40] J. Chu, E. Lu, Y. Chen, X. Leng, Organometallics 32 (2013) 1137.

[41] W.A. Howard, M. Waters, G. Parkin, J. Am. Chem. Soc. 115 (1993) 4917.

[42] W.A. Howard, T.M. Trnka, M. Waters, G. Parkin, J. Organomet. Chem. 528 
(1997) 95.

[43] J.J. Kennedy-Smith, K.A. Nolin, H.P. Gunterman, F.D. Toste, J. Am. Chem. Soc. 125 (2003) 4056.

[44] A.C. Fernandes, R. Fernandes, C.C. Romao, B. Royo, Chem. Commun. (2005) 213.

[45] T.E. Hanna, E. Lobkovsky, P.J. Chirik, Inorg. Chem. 46 (2007) 2359.

[46] S.C.A. Sousa, I. Cabrita, A.C. Fernandes, Chem. Soc. Rev. 41 (2012) 5641. [47] Z.K. Sweeney, J.L. Polse, R.A. Andersen, R.G. Bergman, M.G. Kubinec, J. Am. Chem. Soc. 119 (1997) 4543.

[48] T. Wang, X.Q. Hao, X.X. Zhang, J.F. Gong, M.P. Song, Dalton Trans. 40 (2011) 8964.

[49] E. de Julian, J. Díez, E. Lastra, M.P. Gamasa, J. Mol. Catal. A Chem. 394 (2014) 295.

[50] M. Aydemir, N. Meric, C. Kayan, F. Ok, A. Baysal, Inorganica Chim. Acta 398 (2013) 1.

[51] G. Chelucci, S. Baldino, W. Baratta, Coord.Chem. Rev. 300 (2015) 29.

[52] S. Karabuga, S. Bars, I. Karakaya, S. Gumus, Tetrahedron Lett. 56 (2015) 101.

[53] S.A. Moya, J.C. Araya, J. Gajardo, V. Guerchais, H. Le Bozec, L. Toupet P. Aguirre. Inorg. Chem. Commun, 27 (2013) 108.

[54] P. Aguirre, R. López, D. Villagra, I. Azocar-Guzman, A.J. Pardey, S.A. Moya, Appl. Organomet. Chem. 17 (2003) 36.

[55] S.A. Moya, M. Vidal, K. Brown, C. Negrete-Vergara, G. Abarca, P. Aguirre, Inorg. Chem. Commun. 22 (2012) 146.

[56] J. Gajardo, J.C. Araya, S.A. Moya, A.J. Pardey, V. Guerchais, H. Le Bozec, P. Aguirre, Appl. Organometal. Chem. 20 (2006) 272.

[57] P. Aguirre, R. Sariego, S.A. Moya, J. Coord. Chem. 54 (2001) 323.

[58] D. Oyama, A. Asuma, T. Hamada, T. Takase, Inorg. Chem. Acta 362 (2009) 2581 
[59] P.A Anderson, G. B Deacon, K. H Haarmann, F.R Keene, T.J Meyer, D.A Reitsma, B. W Skelton, G.F Strouse, N.C Thomas, J. A Treadway, A.H White, Inorg. Chem. 1995, 34, 6145-6157

[60] B.G. Harvey, A.M. Arif, R.D. Ernst, B, Polyhedron 23 (2004) 2725.

[61] Piet W.N.M Van Leeuwen "Homogeneous Catalysis" Kluwee Academic, 2004. ISBN 1-4020-2000-7.

[62] I. Jun-Ichi Ito, N. Hisao, Tetrahedron Lett. 55 (2004) 3133. 
Abstract
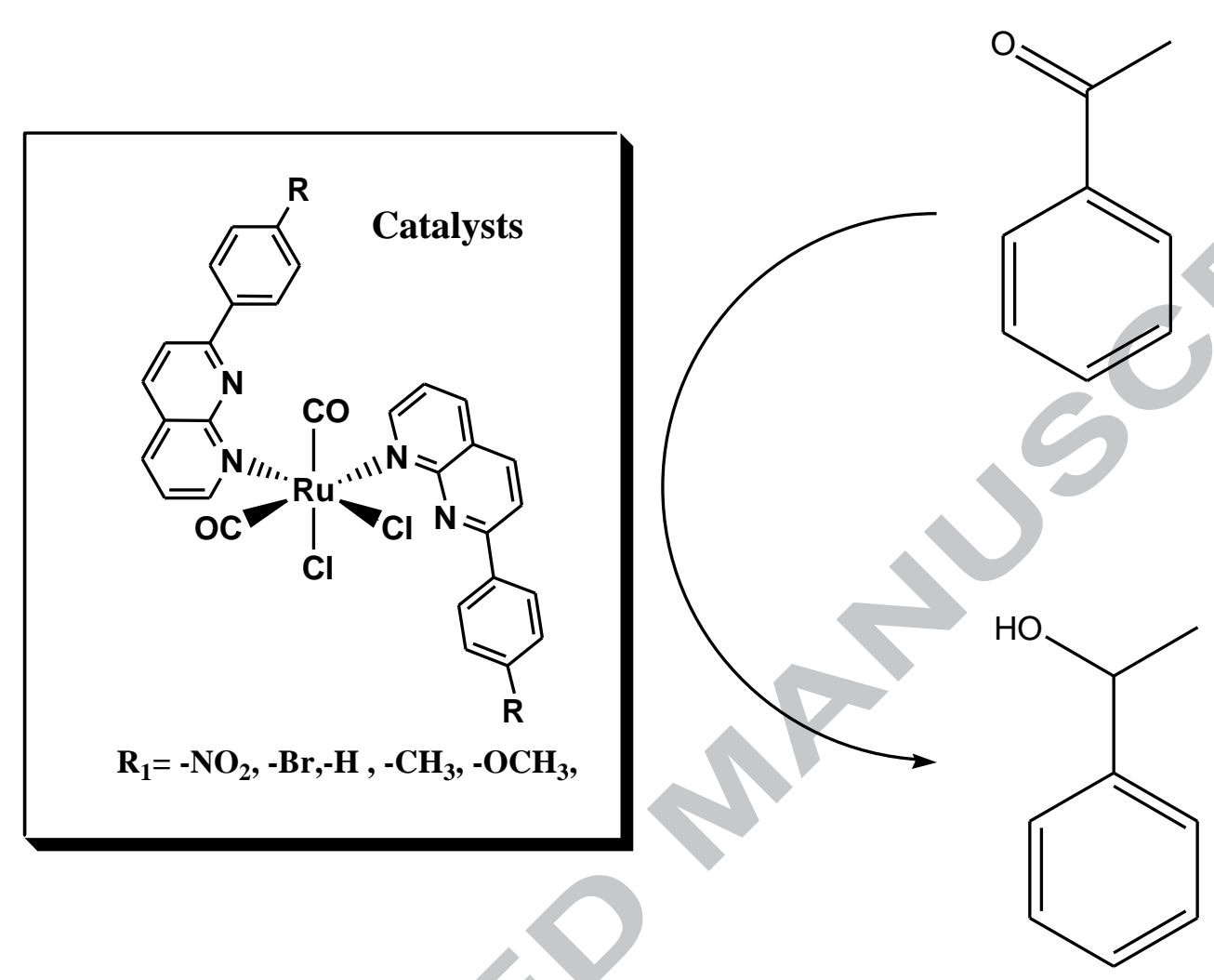
Highlights

1. - Ruthenium complexes containing polypyridine ligands are active catalysts in hydrogen transfer reactions.

2. - The hydrogen transfer reaction is catalyzed by ruthenium 1,8 naphthyridine derivate complexes.

3. - Ruthenium complexes containing 1,8-naphthyridine ligands show high conversions in ketones hydrogenation.

4. - Ruthenium complexes containing polypyridine ligands are alternative catalysts in hydrogenation reactions.

5. - 1,8-Naphthyridine ligands coordinate in a monodentate fashion into the ruthenium monometallic complexes.

6. - The donor properties of the 1,8-naphthyridine ligand stabilize the ruthenium complexes favoring the hydrogenation process. 